\title{
Chemical and Microbiological Attributes of Soil Under Different Management With Application of Swine Wastewater
}

\author{
Maria A. Matos ${ }^{1,2}$, Arnaldo Colozzi Filho ${ }^{2}$, Diva S. Andrade ${ }^{2}$, Graziela M. C. Barbosa ${ }^{2}$, Kelly C. G. P. de Goes ${ }^{2}$, \\ Gisele M. Lovato ${ }^{2}$ \& Marco A. Nogueira ${ }^{1,3}$ \\ ${ }^{1}$ State University of Londrina, Londrina, Paraná, Brazil \\ ${ }^{2}$ Agronomic Institute of Paraná, Londrina, Paraná, Brazil \\ ${ }^{3}$ Brazilian Agricultural Research Corporation, Londrina, Paraná, Brazil \\ Correspondence: Maria A. Matos, State University of Londrina, ZC: 86055-900, Londrina, Paraná, Brazil. Tel: \\ 55-43-3376-2300. E-mail: mariadematos@yahoo.com.br
}

Received: April 25, 2018

doi:10.5539/jas.v10n8p286
Accepted: June 8, 2018 Online Published: July 15, 2018

URL: https://doi.org/10.5539/jas.v10n8p286

This research was financed by the Agronomic Institute of Paraná-IAPAR, Londrina, Paraná, Brazil.

\begin{abstract}
We evaluated the effects of biannual surface applications of swine wastewater (SWW) for 11 years, on chemical and microbiological attributes of a clay-textured soil under no-tillage (NT) or conventional tillage (CT) in Palotina, Paraná, Brazil. The treatments consisted of SWW doses (split into autumn-winter/spring-summer seasons) totaling 30,60, 90 or $120 \mathrm{~m}^{3} \mathrm{ha}^{-1} \mathrm{year}^{-1}$, and a control without application, under CT or NT soil managements (SM). The experimental design was in randomized blocks with a split-plot arrangement and four replications, with SM in the plots and the doses of SWW in the subplots. After 11 years of applications of SWW, soil samples were taken at $0-10,10-20,20-40$, and $40-60 \mathrm{~cm}$ of depth by the end of the winter cover crop, 44 days after the second biannual application. The treatments had only minor effects on the assessed chemical and microbiological soil properties. Available $\mathrm{P}$ and $\mathrm{NO}_{3}{ }^{-}-\mathrm{N}$ increased with the doses of $\mathrm{SWW}$ at the two upper soil layers, whereas NT decreased $\mathrm{NO}_{3}{ }^{-}-\mathrm{N}$ at $10-20 \mathrm{~cm}$ and increased $\mathrm{P}$ at $0-10 \mathrm{~cm} . \mathrm{Zn}^{2+}$ also increased with the doses of SWW, irrespectively the SM. NT increased the microbial biomass C (MBC) at 0-10 cm and 10-20 cm of soil depth, and the microbial quotient $(q \mathrm{Mic})$ at $0-10 \mathrm{~cm}$. SM and SWW did not change the densities of heterotrophic bacteria, fungi, nitrite-oxidizers or ammonifiers at any soil depth. SM had single effects on organic $\mathrm{C}, \mathrm{MBC}$ and $q$ Mic, whereas SWW had single effect only on $\mathrm{Zn}^{2+}$. Only available $\mathrm{P}$ and $\mathrm{NO}_{3}^{-}-\mathrm{N}$ were affected by both SM and SWW. The principal component analysis for chemical and microbiological attributes showed distinct groupings between CT and NT, and SWW rates at $0-10 \mathrm{~cm}$ and $10-20 \mathrm{~cm}$ of soil depths, the most influenced soil layers.
\end{abstract}

Keywords: microbiological indicators, no-tillage, soil fertility, swine residues

\section{Introduction}

The intense swine husbandry activity in southern Brazil generates large amounts of swine wastewater (SWW), which can be used in agricultural soils as a source of nutrients. SWW contains high concentrations of macro and micronutrients and can be used in annual crops and pastures, contributing to the improvement of chemical, physical, and biological soil properties and reducing the costs of agricultural production (Ceretta et al., 2005; Balota et al., 2010; Scherer et al., 2010; Lourenzi et al., 2011).

Organic wastes rich in easily degradable carbon and containing high concentrations of nutrients can influence the soil microbial community and interfere in the cycling processes via biogeochemical cycles of $\mathrm{C}, \mathrm{N}$, and other elements. Most of the $\mathrm{N}$ transformations in the soil are mediated by ammonifying, nitrifying and denitrifying microorganisms. These processes are regulators of $\mathrm{N}$ dynamics in soil and may not only increase $\mathrm{N}$ availability to plants but also favor losses by leaching and denitrification (Paul, 2007).

The soil microbial biomass influences the dynamics of nutrients, increasing nutrient availability via mineralization or decreasing availability via immobilization, affecting the system productivity. The 
immobilization of nutrients in microbial biomass has important implications for the soil-plant system since immobilization protects nutrients from losses and represents an important nutrient reservoir that is easily available (Van Der Heijden et al., 2008).

Microbial biomass carbon (MBC) and other microbiological indicators are important for assessing the effects of the application of residues and soil management (SM). Balota et al. (2012) observed increases in the soil organic $\mathrm{C}$ and MBC with the application of SWW on a clayey soil under conventional tillage (CT) or no tillage (NT). Increases in MBC with the application of pig slurry up to $150 \mathrm{~m}^{3} \mathrm{ha}^{-1}$ year $^{-1}$ in a Typic Haploxeralf were also observed by Plaza et al. (2004). The application of SWW to a clayey-sandy soil increased the number of aerobic heterotrophic bacteria by 1.3 (Suresh et al., 2009). Soil conservation managements also favors the MBC, as indicated by increases in MBC under NT compared with CT (Mercante et al., 2008; Balota et al., 2012).

Soil applications of SWW brings not only organic $\mathrm{C}$ but also several macro and micronutrients, such as $\mathrm{Cu}^{2+}$ and $\mathrm{Zn}^{2+}$, which excess may impair the microbial community and their processes in soil. For example, the accumulation of trace elements because of successive applications of SWW to agricultural soil was associated to negative effects on soil microbial biomass and activity (Dias-Júnior et al., 1998; Renella et al., 2007; Balota et al., 2011). The addition of $\mathrm{Cd}$ and $\mathrm{Zn}$ also reduced the number of rhizobia in native clover but did not influence the soil microbial biomass (Renella et al., 2007). Furthermore, ammonifying microorganisms decreased by $67 \%$ and $19 \%$ with the addition of SWW at rates of 90 and $120 \mathrm{~m}^{3} \mathrm{ha}^{-1}$ year ${ }^{-1}$, respectively (Lalande et al., 2000). Research on the effects of SWW application on microbiological attributes under different SM strategies is relevant to advice practices to protect and improve the quality of agricultural soils.

The objective of this work was to evaluate the effects of application of SWW for 11 consecutive years on chemical and microbiological attributes of a clayey soil managed under CT and NT in southern Brazil.

\section{Material and Methods}

\subsection{Description of the Area and Experimental Design}

The experiment was installed in 1996 in the municipality of Palotina, Paraná, Brazil $\left(24^{\circ} 18^{\prime} 50.47^{\prime \prime} \mathrm{S}\right.$ and $53^{\circ} 54^{\prime} 13.35^{\prime \prime} \mathrm{W}, 308 \mathrm{~m}$ above sea level and slope between $0.5 \%$ and $3 \%$ ). The local climate is humid subtropical (Cfa) according to Koeppen's classification. The soil is classified as Typic Hapludox (Soil Survey Staff, 2015), equivalent to "Latossolo Vermelho distroférrico" based on the Brazilian classification (Santos et al., 2013), with clayey texture $(60 \%$ clay, $24 \%$ sand and $16 \%$ silt). The treatments consisted of biannual surface applications of SWW totaling 30,60, 90 and $120 \mathrm{~m}^{3} \mathrm{ha}^{-1}$ year $^{-1}$, and a control without application, under CT or NT soil managements. The soil was cropped with rotations of soybean/wheat (Glycine max (L.) Merrill/Triticuma estivum); corn/oats+vetch (Zea mays L./Avena sativa+Vicia sativa); and soybean/turnip (Raphanus sativus) in the spring-summer/autumn-winter seasons, respectively.

The experimental design consisted of randomized blocks with split-plot arrangement in four replications separated by 15 to $20 \mathrm{~m}$ corridors to avoid cross contaminations. Each plot had $500 \mathrm{~m}^{2}(50 \mathrm{~m} \times 10 \mathrm{~m})$ split into two SM systems, CT or NT, each with $50 \mathrm{~m} \times 5 \mathrm{~m}$. The SWW treatments were applied in the subplots $(10 \mathrm{~m} \times 5$ $\mathrm{m})$ at the corresponding rates, split two times a year, $50 \%$ at the beginning of the spring-summer crop and $50 \%$ at the beginning of the autumn-winter crop.

The SWW was collected after 4 to 5 months of stabilization from waste aerobic or maturation pools of swine husbandry farms close to the experimental site and analyzed for physicochemical properties (Table 1). Based on the concentrations, the total annual inputs to the soil was estimated. On November 7 of the eleventh year of the experiment, after the cultivation of turnips, SWW was applied on the soil surface, and subsequently incorporated in the plots under CT with a disc plow $(0.2 \mathrm{~m}$ depth) followed by harrowing $(0.1 \mathrm{~m} \mathrm{depth})$, or left on the soil surface in the plots under NT. On November 13, all plots were sown with soybean. 
Table 1. Physicochemical characteristics of the SWW applied in the experimental site and annual inputs provided by each dose

\begin{tabular}{|c|c|c|c|c|c|c|c|c|c|c|}
\hline & $\mathrm{N}$ & $\mathrm{P}$ & $\mathrm{K}^{+}$ & $\mathrm{Ca}^{2+}$ & $\mathrm{Mg}^{2+}$ & $\mathrm{Cu}^{2+}$ & $\mathrm{Zn}^{2+}$ & $\mathrm{Mn}^{2+}$ & Total solids & $\mathrm{pH}$ \\
\hline \multirow{2}{*}{ SWW composition ${ }^{1}$} & -- & & $-\cdots$ & - & - & $-1-$ & - & $\cdots$ & $----\cdot-$ & \multirow[b]{2}{*}{6.8} \\
\hline & 3.3 & 3.2 & 4.7 & 3.4 & 1.5 & 0.05 & 0.10 & 0.05 & 11.90 & \\
\hline Dose, $m^{3} h a^{-1} y r^{-1}$ & \multicolumn{9}{|c|}{ Total inputs, $\mathrm{kg} \mathrm{ha}^{-1} \mathrm{yr}^{-1}{ }^{-1}$} & \\
\hline 30 & 99 & 96 & 141 & 102 & 45 & 1.5 & 3 & 1.5 & 357 & - \\
\hline 60 & 198 & 192 & 282 & 204 & 90 & 3.0 & 6 & 3.0 & 714 & - \\
\hline 90 & 297 & 288 & 423 & 306 & 135 & 4.5 & 9 & 4.5 & 1071 & - \\
\hline 120 & 396 & 384 & 564 & 408 & 180 & 6.0 & 12 & 6.0 & 1428 & - \\
\hline
\end{tabular}

Note. ${ }^{1}$ Total contents.

\subsection{Soil Sampling, and Chemical and Microbiological Analyses}

Soil samples were taken after 11 consecutive years of SWW application in both SM systems. Forty-four days after the last application of SWW, samples formed by 3 subsamples were collected with a cutting blade at $0-10$, $10-20 \mathrm{~cm}, 20-40 \mathrm{~cm}$ and $40-60 \mathrm{~cm}$ and sieved $(2 \mathrm{~mm})$. In air-dried samples, the soil chemical analyses were performed according to Pavan et al. (1992); $\mathrm{Cu}^{2+}$ and $\mathrm{Zn}^{2+}$ were determined by inductively coupled plasma (ICP) mass spectrometry (Silva et al., 2009). Soil organic matter (SOM) was determined by incineration in muffle furnace at $600{ }^{\circ} \mathrm{C}$ for $6 \mathrm{~h}$ (Goldin, 1987), whereas total organic carbon (TOC) was determined by the Walkley-Black method. Nitrate $\left(\mathrm{NO}_{3}^{-}-\mathrm{N}\right)$ and ammonium $\left(\mathrm{NH}_{4}{ }^{-}-\mathrm{N}\right)$ were determined according to Miyazawa et al. (1985), and Kempers and Zweers (1986), respectively.

For microbiological analyses, soil samples were stored at $5{ }^{\circ} \mathrm{C}$ at field moisture. The ammonifying and nitrite-oxidizing microorganisms were estimated by the most probable number (MPN) method (Andrade et al., 1994). Culturable bacteria and fungi were estimated by the MPN based on the droplet-plating method (Jahnel et al., 1999) in nutrient agar for bacteria and Martin's medium for fungi. Microbial Biomass Carbon (MBC) was estimated by the fumigation-extraction method (Vance et al., 1987) using the correction factor $\mathrm{K}_{\mathrm{c}}=0.33$ (Sparling \& West, 1988). The microbial quotient $(q \mathrm{Mic})$ was calculated as $q \mathrm{Mic}=(\mathrm{MBC} / \mathrm{TOC}) \times 100($ Sparling, 1992).

\subsection{Statistical Analysis}

The MPN estimated for total bacteria, fungi, ammonifiers and nitrite oxidizers were log-transformed before analysis. Analysis of variance ( $F$ test, $p \leq 0.05)$ followed comparison of means by Tukey's test $(p \leq 0.05)$ with the software SISVAR (Ferreira, 2011). Principal component analysis was applied to the dataset to evaluate the relationship of chemical and microbiological variables with the doses of SWW and SM for each soil depth, using the software XLSTAT Base (Addinsoft, 2009).

\section{Results}

Soil management (SM) had significant influence on P concentration at $0-10 \mathrm{~cm}$ and $10-20 \mathrm{~cm}$ and on the concentrations of TOC and $\mathrm{NO}_{3}^{-}-\mathrm{N}$ at 10-20 cm (Table 2). SM and SWW applications did not change the soil pH, $\mathrm{SOM}, \mathrm{H}+\mathrm{Al}, \mathrm{Ca}^{2+}, \mathrm{Mg}^{2+}$ and $\mathrm{K}^{+}$at any soil depth. The applications of $\mathrm{SWW}$, in each $\mathrm{SM}$, increased the availability of $\mathrm{P}$ and $\mathrm{NO}_{3}^{-}-\mathrm{N}$ at $0-10 \mathrm{~cm}$ and $10-20 \mathrm{~cm}$, and $\mathrm{Zn}^{2+}$ at $10-20 \mathrm{~cm}$, independently of the SM (not shown). NT increased the concentration of $\mathrm{P}$ at $0-10 \mathrm{~cm}$, whereas decreased $\mathrm{NO}_{3}^{-}-\mathrm{N}$ at $10-20 \mathrm{~cm}$. The $\mathrm{P}$ concentrations in the soil profile were higher at $0-10 \mathrm{~cm}$ and $10-20 \mathrm{~cm}$ and decreased by up to $93 \%$ in the following depths (Table 2).

NT significantly increased MBC at $0-10$ and $10-20 \mathrm{~cm}$ of soil depth as well as $q$ Mic at $0-10 \mathrm{~cm}$ (Table 3). There was no significant effect on culturable bacteria, fungi, nitrite oxidizers, and ammonifiers at any depth. No interaction was detected between SM and rates of SWW application on the microbiological variables at any soil depth (Table 3).

Based on chemical (12) and microbiological (6) attributes, PCA discriminated SWW doses and more clearly the $\mathrm{CT}$ and NT soil managements at the two topsoil layers (Figure 1). At $0-10 \mathrm{~cm}$, the principal components F1 (42.6\%) and F2 (21.6\%) explained $64.3 \%$ of the total variance, where NT was mainly associated to the positive side of F1, whereas CT was restricted to the negative side. The doses of SWW were quite well organized in two distinct groupings along F1 (Figure 1). Most of the variables were associated with NT, whereas only few variables (e.g., Bac, Nit-ox, and $\mathrm{Cu}^{2+}$ ) were associated with CT (Figure 1). 
At $10-20 \mathrm{~cm}$, the components F1 (31.2\%) and F2 (22.9\%) explained $54.2 \%$ of the total variance. The soil managements CT and NT grouped more distinctly and SWW followed an organization clearly defined by the doses within each SM. CT predominated in the positive side of the F1 axis, and was associated with Fungi, Nit-ox, C, Bac, $\mathrm{NH}_{4}{ }^{-}-\mathrm{N}, \mathrm{NO}_{3}^{-}-\mathrm{N}$, and $\mathrm{Zn}^{2+}$ (Figure 1). On the other hand, NT positioned on the negative side of F1 axis, and was associated with MBC, $q$ Mic, and ammonifiers; $\mathrm{SOM}$ and $\mathrm{Mg}^{2+}$ were associated to higher doses of SWW under NT along F2 axis (Figure 1).

At $20-40 \mathrm{~cm}$, the components F1 (32.7\%) and F2 (19.4\%) explained $52.4 \%$ of the total variance, but the treatments were not clearly separated as before (Figure 1). Only few treatments were clearly associated to the variables, like the dose $120 \mathrm{~m}^{-3} \mathrm{ha}^{-1} \mathrm{yr}^{-1}$ at both $\mathrm{CT}$ and $\mathrm{NT}$ associated with $\mathrm{NH}_{4}{ }^{+}-\mathrm{N}$ and $\mathrm{P}$ along the positive side of axis F1. MBC, fungal density, and $q$ Mic were associated in the negative side with NT and CT without SWW.

At $40-60 \mathrm{~cm}$, the components F1 (24.5\%) and F2 (20.3\%) explained $44.8 \%$ of variance (Figure 1). Similarly to $20-40 \mathrm{~cm}$, there was no clear separation of the treatments. The control and $120 \mathrm{~m}^{-3} \mathrm{ha}^{-1} \mathrm{yr}^{-1}$ of SWW posed oppositely each other and grouped within the same SM. P, TOC, $\mathrm{Zn}^{2+}, \mathrm{NO}_{3}{ }^{-}-\mathrm{N}$, Ammonifyiers, Nitrite oxidizers, MBC, $\mathrm{Ca}^{2+}$ and SOM were associated with $120 \mathrm{~m}^{-3} \mathrm{ha}^{-1} \mathrm{yr}^{-1}$ of SWW, irrespectively the SM.

Table 2. Physicochemical attributes at four depths of a clayey soil (Typic Hapludox) after applications of doses of swine wastewater (SWW) for 11 years under conventional tillage (CT) or no-tillage (NT) in southern Brazil

\begin{tabular}{|c|c|c|c|c|c|c|c|c|c|c|c|c|c|c|c|c|c|c|c|c|c|c|c|c|}
\hline \multirow{2}{*}{$\begin{array}{l}\text { SWW } \\
\text { dose }\end{array}$} & \multicolumn{2}{|c|}{$\mathrm{P}$} & \multicolumn{2}{|c|}{ TOC } & \multicolumn{2}{|c|}{ SOM } & \multicolumn{2}{|c|}{$\mathrm{pH}^{2}$} & \multicolumn{2}{|c|}{$\mathrm{H}+\mathrm{AL}$} & \multicolumn{2}{|c|}{$\mathrm{Ca}^{2+}$} & \multicolumn{2}{|c|}{$\mathrm{Mg}^{2+}$} & \multicolumn{2}{|c|}{$\mathrm{K}^{+}$} & \multicolumn{2}{|c|}{$\mathrm{NO}_{3}^{-}$} & \multicolumn{2}{|c|}{$\mathrm{NH}_{4}^{+}$} & \multicolumn{2}{|c|}{$\mathrm{Cu}^{2+}$} & \multicolumn{2}{|c|}{$\mathrm{Zn}^{2+}$} \\
\hline & $\mathrm{CT}$ & NT & $\mathrm{T}$ & NT & $\mathrm{T}$ & NT & $\mathrm{CT}$ & JT & $\mathrm{CT}$ & NT & $\mathrm{CT}$ & NT & $\mathrm{CT}$ & NT & CT & NT & $\mathrm{CT}$ & NT & $\mathrm{CT}$ & NT & $\mathrm{CT}$ & NT & $\mathrm{CT}$ & NT \\
\hline \multicolumn{9}{|c|}{$\mathrm{m}^{3} \mathrm{ha}^{-1} \mathrm{yr}^{-1}$-- $\mathrm{mg} \mathrm{dm}^{-3}$} & \multicolumn{8}{|c|}{ - } & \multicolumn{8}{|c|}{ 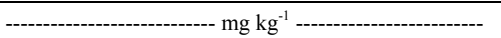 } \\
\hline \multicolumn{25}{|c|}{ Depth $0-10 \mathrm{~cm}$} \\
\hline 0 & $10.4 \mathrm{c}$ & $13.0 \mathrm{c}$ & $15.0^{\mathrm{ns}}$ & $15.8^{\text {ns }}$ & $40.0^{\mathrm{ns}}$ & $43.8^{\text {ns }}$ & $4.9^{\text {ns }}$ & $4.9^{\mathrm{ns}}$ & $5.1^{\mathrm{ns}}$ & $5.1^{\mathrm{ns}}$ & $4.5^{\mathrm{ns}}$ & $4.6^{\mathrm{ns}}$ & $1.5^{\mathrm{ns}}$ & $1.7^{\mathrm{ns}}$ & $0.83^{\mathrm{ns}}$ & $1.1^{\mathrm{ns}}$ & $23.9 \mathrm{~b}$ & $22.3 \mathrm{~b}$ & $6.5^{\mathrm{ns}}$ & $6.7^{\mathrm{ns}}$ & $11.2^{\mathrm{ns}}$ & $10.5^{\mathrm{ns}}$ & $7.0^{\mathrm{ns}}$ & $6.8^{\mathrm{ns}}$ \\
\hline 30 & $2.1 b c$ & $44.1 \mathrm{~b}$ & 14.7 & 15.9 & 40.3 & 44.3 & 4.9 & 5.0 & 5.1 & 5.3 & 4.4 & 4.9 & 1.7 & 1.9 & 0.96 & 1.1 & $34.4 \mathrm{ab}$ & $24.5 \mathrm{ab}$ & 7.0 & 8.2 & 11.8 & 9.6 & 7.8 & 9.0 \\
\hline 60 & $.4 a b c$ & $44.5 \mathrm{~b}$ & 5.7 & 15.2 & 41.2 & 42.6 & 4.8 & 4.7 & 5.3 & 5.7 & 4.6 & 4 & 1.7 & 1.7 & 1.01 & 1.0 & $31.9 \mathrm{ab}$ & $29.2 \mathrm{ab}$ & 6.7 & 8.2 & 10.7 & 10.9 & 9 & 9.5 \\
\hline 90 & $0.9 \mathrm{ab}$ & $5.6 \mathrm{a}$ & 15.2 & 14 & 41.1 & 4 & 4. & 4 & 5 & 6.0 & 4 & 4.2 & 1 & .6 & 0 & 1.0 & $32.9 \mathrm{ab}$ & 3 & .2 & 7.9 & 10.6 & 12.4 & 8.2 & 9.8 \\
\hline 120 & $2.8 \mathrm{a}$ & $78.3 \mathrm{a}$ & 16.1 & 16.8 & 42.2 & 45.6 & 4.8 & 4.7 & 5.7 & 6.0 & 4.7 & 4.5 & 1.7 & 1.9 & 0.97 & 1.1 & $38.4 \mathrm{a}$ & $34.9 \mathrm{a}$ & 7.3 & 8.5 & 11.9 & 10.3 & 9.3 & 11.4 \\
\hline Mea & $28.7 \mathrm{~B}$ & $49.1 \mathrm{~A}$ & $15.3^{\text {ns }}$ & 15.8 & $41.0^{\mathrm{ns}}$ & 43.8 & $4.9^{\text {ns }}$ & 4.8 & $5.3^{\text {ns }}$ & 5.6 & $4.5^{\mathrm{ns}}$ & 4.4 & $1.7^{\text {ns }}$ & 1.7 & & 1.1 & $32.3^{\text {ns }}$ & 29.0 & $6.9^{\mathrm{ns}}$ & 7.9 & $11.2^{\mathrm{ns}}$ & 10.8 & $8.329^{\text {ns }}$ & 9.3 \\
\hline 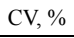 & 18.6 & 23.2 & 7.9 & 8.0 & 11.8 & 10.2 & 2.0 & 5.8 & 8.3 & 12.0 & 23.6 & 11.4 & 11.3 & 33.6 & 25.767 & 17.0 & 17.1 & 32.3 & 32.3 & 14.6 & 18.7 & 16.2 & 26 & 32.6 \\
\hline \multicolumn{25}{|c|}{ Depth $10-20 \mathrm{~cm}$} \\
\hline 0 & $10.1 \mathrm{c}$ & $6.4 \mathrm{c}$ & $13.3^{\text {ns }}$ & $15.5^{\mathrm{ns}}$ & $38.9^{\text {ns }}$ & $39.7^{\text {ns }}$ & $5.1^{\mathrm{ns}}$ & $5.7^{\mathrm{ns}}$ & $4.9^{\mathrm{ns}}$ & $4.5^{\mathrm{ns}}$ & $4.9^{\text {ns }}$ & $4.8^{\mathrm{ns}}$ & $1.6^{\mathrm{ns}}$ & $1.5^{\mathrm{ns}}$ & $0.9^{\text {ns }}$ & $0.9^{\mathrm{ns}}$ & $25.2 b$ & $16.6 \mathrm{~b}$ & $6.8^{\text {ns }}$ & $5.6^{\mathrm{ns}}$ & $11.9^{\text {ns }}$ & $12.1^{\mathrm{ns}}$ & $4.8^{\mathrm{ns}}$ & $4.1^{\mathrm{ns}}$ \\
\hline 3( & $19.8 \mathrm{bc}$ & $11.5 \mathrm{c}$ & 14.4 & 14.8 & 39.4 & 41.9 & 5.7 & 6.0 & 4.8 & 4.3 & 4.7 & 5.3 & 1.7 & 1.8 & 1.0 & 1.0 & $32.9 \mathrm{ab}$ & $18.2 \mathrm{ab}$ & 6.9 & 5.8 & 12.1 & 12.0 & 5.5 & 4.2 \\
\hline 60 & $24.7 \mathrm{~b}$ & $12.6 \mathrm{bc}$ & 13.1 & 14.8 & 39.8 & 40.0 & 5.2 & 5.8 & 5.4 & 5.2 & 4.4 & 4.3 & 1.6 & 1.5 & 0.9 & 1.0 & $31.6 \mathrm{ab}$ & $21.8 \mathrm{ab}$ & 6.9 & 6.6 & 12.3 & 11.1 & 5.6 & 4.1 \\
\hline 90 & $27.1 \mathrm{ab}$ & $22.7 \mathrm{ab}$ & 12.1 & 14.3 & 38.5 & 38.6 & 5.0 & 5.3 & 5.5 & 5.5 & 4.6 & 4.5 & 1.6 & 1.7 & 0 . & 0.9 & $32.1 \mathrm{ab}$ & $25.9 \mathrm{ab}$ & 6.6 & 6.8 & 10.4 & 11.4 & 6 & 5.1 \\
\hline 120 & 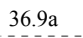 & 24 & 0.1 & 1 & 42.9 & 43.8 & 5.6 & 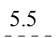 & 5.6 & 5.4 & 5.2 & 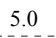 & 1.7 & . & 1.1 & 1 & $39.0 \mathrm{a}$ & $\mathrm{a}$ & 7.0 & 6.7 & 12.1 & 11.9 & 7.1 & 5.7 \\
\hline $\mathrm{M}$ & $23.7 \mathrm{~A}$ & $15.6 \mathrm{~B}$ & $13.3 \mathrm{~B}$ & $15.1 \mathrm{~A}$ & $39.9^{\text {ns }}$ & 40.8 & $5.3^{\text {ns }}$ & 5.7 & $5.2^{\text {ns }}$ & 5.0 & $4.8^{\mathrm{ns}}$ & 4.8 & $1.6^{\mathrm{ns}}$ & 1.7 & 1 & 1.0 & A & $22.0 \mathrm{~B}$ & $6.8^{\mathrm{ns}}$ & 6.3 & $11.7^{15}$ & 11.7 & 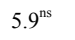 & 4.6 \\
\hline $\mathrm{V}, \%$ & 23.0 & 25.2 & 11.2 & 11.9 & 12.7 & 9.6 & 4.0 & 5.3 & 8.9 & 12.5 & 11.7 & 17.7 & 16.0 & 12.9 & 25.6 & 21.7 & 24.1 & 16.7 & 11.5 & 11.3 & 8.9 & 16.7 & 25.6 & 21.1 \\
\hline \multicolumn{25}{|l|}{ Dept } \\
\hline 0 & 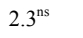 & $2.0^{\mathrm{ns}}$ & $7.3^{\mathrm{ns}}$ & $7.1^{\mathrm{ns}}$ & $27.2^{\mathrm{ns}}$ & $30.8^{\text {ns }}$ & $5.4^{\mathrm{ns}}$ & $5.4^{\mathrm{ns}}$ & $3.6^{\mathrm{ns}}$ & $3.5^{\mathrm{ns}}$ & $5.5^{\mathrm{ns}}$ & $5.3^{\mathrm{ns}}$ & $1.6^{\mathrm{ns}}$ & $1.6^{\mathrm{ns}}$ & $0.6^{\mathrm{ns}}$ & $0.6^{\mathrm{ns}}$ & $18.7 \mathrm{~b}$ & $\mathrm{~b}$ & $7.0^{\mathrm{ns}}$ & $7.0^{\mathrm{ns}}$ & $10.7^{\mathrm{ns}}$ & $10.1^{\mathrm{ns}}$ & $2.2^{\mathrm{ns}}$ & $2.0^{\mathrm{ns}}$ \\
\hline 30 & & 2 & 7 & 7 & 28.0 & 3 & 5 & 5 & 3 & 3 & 5 & 5.2 & 1.7 & 1.6 & 0.6 & 0.6 & $a b$ & $\mathrm{~b}$ & 7 & 1 & 10.5 & 12.2 & & 2.0 \\
\hline 60 & & 2 & 7 & 6.6 & 27.5 & 29.9 & 5 & $\mathrm{~J}$ & 3 & 3 & 4 & 5.0 & 1.7 & 1.6 & 0.6 & 0 & $a b$ & $\mathrm{~b}$ & 7.2 & 0 & 11.8 & 6 & & 7 \\
\hline 90 & 2.8 & 2.8 & 7.0 & 6.8 & 28.7 & 29.0 & 5.4 & 5.0 & 3.0 & 3.9 & 5.1 & 4.0 & 1.0 & 1.1 & 0. & 0.0 & $28.6 \mathrm{ab}$ & $23.9 \mathrm{ab}$ & 6.5 & 7.1 & 10.4 & 11.3 & 2.0 & 2.5 \\
\hline 120 & 3.5 & 3.6 & 7.3 & $\begin{array}{r}7.3 \\
-\end{array}$ & 31.1 & 31.5 & 5.4 & 5.3 & 3.7 & 3.6 & 5.0 & 5.0 & 1.7 & 1.6 & 0.6 & 0.6 & $30.6 \mathrm{a}$ & $25.4 \mathrm{a}$ & 7.5 & 7.6 & 11.0 & 9.9 & 2.4 & 2.6 \\
\hline 101 & $6^{\text {ns }}$ & 2.1 & $7.3^{\mathrm{ns}}$ & 7.1 & $28.5^{\text {ns }}$ & 30.5 & $5.3^{\text {ns }}$ & 5.3 & $3.6^{\mathrm{ns}}$ & 3.5 & $5.2^{\mathrm{ns}}$ & 5.0 & $1.7^{\text {ns }}$ & 1.6 & $0.60^{\mathrm{ns}}$ & 0.6 & $25.3^{\text {ns }}$ & 17.0 & $7.1^{\mathrm{ns}}$ & 7.2 & $10.9^{\text {ns }}$ & 10.8 & $2.3^{\mathrm{ns}}$ & 2.2 \\
\hline $\mathrm{CV}, \%$ & 17 & 36.1 & , & 15.4 & 12.3 & 10.5 & 2.8 & 6.7 & 10.0 & 13.1 & 25.4 & 24.4 & 14.6 & 10.6 & 25.9 & 31.2 & 3 & 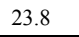 & .3 & 14.7 & 22.1 & 16.4 & 31.4 & 20. \\
\hline Depth & $0 \mathrm{~cm}$ & & & & & & & & & & & & & & & & & & & & & & & \\
\hline 0 & $2.6^{\mathrm{ns}}$ & $2.2^{\mathrm{ns}}$ & $6.9^{\mathrm{ns}}$ & $6.9^{\mathrm{ns}}$ & $27.4^{\mathrm{ns}}$ & $30.3^{\text {ns }}$ & $5.5^{\mathrm{ns}}$ & $5.4^{\mathrm{ns}}$ & $3.4^{\mathrm{ns}}$ & $3.5^{\mathrm{ns}}$ & $4.9^{\mathrm{ns}}$ & $4.6^{\mathrm{ns}}$ & $1.5^{\mathrm{ns}}$ & $1.4^{\mathrm{ns}}$ & $0.4^{\mathrm{ns}}$ & $0.4^{\mathrm{ns}}$ & $17.0^{\mathrm{ns}}$ & $12.8 \mathrm{~b}$ & $6.8^{\mathrm{ns}}$ & $6.7^{\mathrm{ns}}$ & $8.7^{\mathrm{ns}}$ & $9.3^{\mathrm{ns}}$ & $1.6^{\mathrm{ns}}$ & $1.8^{\mathrm{ns}}$ \\
\hline 30 & 25 & 2.6 & 7.0 & 7.1 & 28.3 & 30.5 & 5.4 & 5.6 & 3.4 & 3.1 & 4.7 & 4.8 & 1.7 & 1.6 & 0.4 & 0.5 & 19.8 & $16.4 \mathrm{ab}$ & 7.1 & 6.5 & 8.1 & .8 & 17 & 1.7 \\
\hline 66 & 2.6 & 2 & 6 & 6. & 25.2 & 28.1 & 5.4 & 5.6 & 3.4 & 3.1 & 4.5 & 4.9 & 1.6 & 1.5 & 0 & 0.4 & 1 & $\mathrm{ab}$ & 6.5 & 6.8 & 9 & 1 & 1.8 & 2.0 \\
\hline 90 & 3.0 & 2.3 & 7.1 & 5.9 & 27.1 & 27.3 & 5.5 & 5.2 & 3.5 & 3.4 & 4.4 & 4.3 & 1.7 & 1.6 & 0. & 0.4 & 9 & $17.8 \mathrm{ab}$ & 7.0 & 6.6 & 9.0 &.$J$ & 2.0 & 1.9 \\
\hline 120 & 3.2 & 2.8 & 7.2 & 7.1 & 29.8 & 29.6 & 5.4 & 5.5 & 3.4 & 3.3 & 4.9 & 4.9 & 1.6 & 1.5 & 0.4 & 0.4 & 25.5 & $23.6 \mathrm{a}$ & 6.5 & 6.6 & 9.8 & 8.8 & 2.1 & 1.9 \\
\hline Mean & $2.7^{\mathrm{ns}}$ & 2.5 & $6.9^{\mathrm{ns}}$ & 6.61 & $27.5^{\mathrm{ns}}$ & 29.2 & $5.5^{\mathrm{ns}}$ & 5.5 & $3.4^{\mathrm{ns}}$ & 3.3 & $4.7^{\mathrm{n}}$ & 4.7 & $1.6^{\mathrm{ns}}$ & 1.5 & $0.4^{\mathrm{ns}}$ & 0.4 & $20.8^{\mathrm{ns}}$ & 17.3 & $6.8^{\text {ns }}$ & 6.6 & $9.0^{\mathrm{ns}}$ & 9.5 & $1.8^{\mathrm{ns}}$ & 1.9 \\
\hline $\mathrm{CV}, \%$ & 16.1 & 18.7 & 7.4 & 17.3 & 18.7 & 12.8 & 3.7 & 6.5 & 10.0 & 10.2 & 17.8 & 21.1 & 14.9 & 10.7 & 11.2 & 34.7 & 21.9 & 25.7 & 30.1 & 11.5 & 22.1 & 18.2 & 20.9 & 36.4 \\
\hline
\end{tabular}

Note. Means followed by the same letter, small in column, capital in line, do not differ each other by the Tukey's test at $\mathrm{p} \leq 0.05 ; \mathrm{ns}=$ not significant by the $\mathrm{F}$ test $(\mathrm{p} \leq 0.05)$. $\mathrm{P}$ and $\mathrm{K}^{+}$extracted with Mehlich-1; $\mathrm{pH}$ in $\mathrm{CaCl}_{2}$, $0.01 \mathrm{~mol} \mathrm{~L}^{-1} ; \mathrm{Ca}^{2+}$ and $\mathrm{Mg}^{2+}$ extracted with $\mathrm{KCl} 1 \mathrm{~mol} \mathrm{~L}^{-1} ; \mathrm{H}+\mathrm{Al}$ determined in SMP buffer; $\mathrm{TOC}=$ total organic carbon determined by the Walkley-Black method; $\mathrm{SOM}=$ soil organic matter, determined by the muffle furnace method; $\mathrm{Cu}^{2+}$ and $\mathrm{Zn}^{2+}$ extracted with $\mathrm{HCl} 0.1 \mathrm{~mol} \mathrm{~L}^{-1} ; \mathrm{NO}_{3}^{-}$and $\mathrm{NH}_{4}^{+}$extracted with $\mathrm{KCl} 2 \mathrm{~mol} \mathrm{~L}^{-1}$; $\mathrm{CV}=$ coefficient of variation. 
Table 3. Microbiological attributes at four depths of a clayey soil (Typic Hapludox) after applications of doses of swine wastewater (SWW) for 11 years under conventional tillage (CT) or no-tillage (NT) in southern Brazil

\begin{tabular}{|c|c|c|c|c|c|c|c|c|c|c|c|c|}
\hline \multirow{2}{*}{ SWW dose } & \multicolumn{2}{|c|}{$\mathrm{MBC}^{1}$} & \multicolumn{2}{|c|}{$q \mathrm{Mic}^{2}$} & \multicolumn{2}{|c|}{ Bacteria } & \multicolumn{2}{|c|}{ Fungi } & \multicolumn{2}{|c|}{ Nitrite oxidizers $^{3}$} & \multicolumn{2}{|c|}{ Ammonifiers $^{3}$} \\
\hline & CT & NT & $\mathrm{CT}$ & NT & $\mathrm{CT}$ & NT & $\mathrm{CT}$ & NT & $\mathrm{CT}$ & NT & $\mathrm{CT}$ & NT \\
\hline$-\mathrm{m}^{3} \mathrm{ha}^{-1} \mathrm{yr}^{-1}--$ & \multicolumn{2}{|c|}{------ $\mu \mathrm{g} \mathrm{g}^{-1}$} & \multirow{2}{*}{\multicolumn{2}{|c|}{------- \% ------ }} & \multicolumn{8}{|c|}{ - } \\
\hline \multicolumn{12}{|l|}{ Depth $0-10 \mathrm{~cm}$} & \\
\hline 0 & $160.5^{\mathrm{ns}}$ & $247.9^{\mathrm{ns}}$ & $1.1^{\mathrm{ns}}$ & $1.6^{\mathrm{ns}}$ & $6.2^{\text {ns }}$ & $6.1^{\mathrm{ns}}$ & $5.1^{\mathrm{ns}}$ & $4.9^{\mathrm{ns}}$ & $5.8^{\mathrm{ns}}$ & $5.5^{\mathrm{ns}}$ & $7.3^{\mathrm{ns}}$ & $7.4^{\mathrm{ns}}$ \\
\hline 30 & 185.6 & 266.4 & 1.3 & 1.7 & 6.2 & 6.1 & 5.0 & 5.5 & 6.1 & 4.7 & 7.7 & 7.6 \\
\hline 60 & 185.2 & 280.9 & 1.2 & 1.8 & 6.1 & 6.4 & 5.6 & 5.3 & 5.8 & 5.7 & 7.4 & 7.7 \\
\hline 90 & 190.9 & 262.7 & 1.3 & 1.7 & 5.9 & 5.8 & 4.7 & 5.3 & 5.5 & 5.5 & 8.0 & 7.7 \\
\hline 120 & 184.9 & 273.6 & 1.1 & 1.6 & 6.1 & 5.9 & 5.2 & 5.4 & 5.1 & 5.7 & 7.3 & 7.3 \\
\hline Mean & $181.4 \mathrm{~B}$ & $266.3 \mathrm{~A}$ & $1.2 \mathrm{~B}$ & $1.7 \mathrm{~A}$ & $6.1^{\text {ns }}$ & 6.1 & $5.1^{\mathrm{ns}}$ & 5.3 & $5.7^{\mathrm{ns}}$ & 5.4 & $7.5^{\mathrm{ns}}$ & 7.5 \\
\hline $\mathrm{CV}, \%$ & 32.0 & 14.5 & 36.8 & 17.0 & 4.1 & 6.9 & 6.9 & 7.1 & 7.2 & 7.4 & 8.7 & 9.0 \\
\hline \multicolumn{13}{|l|}{ Depth $10-20 \mathrm{~cm}$} \\
\hline 0 & $107.0^{\mathrm{ns}}$ & $150.9^{\mathrm{ns}}$ & $0.7^{\mathrm{ns}}$ & $1.1^{\mathrm{ns}}$ & $5.8^{\mathrm{ns}}$ & $5.5^{\mathrm{ns}}$ & $4.0^{\mathrm{ns}}$ & $3.6^{\mathrm{ns}}$ & $5.1^{\mathrm{ns}}$ & $5.6^{\mathrm{ns}}$ & $6.5^{\mathrm{ns}}$ & $7.1^{\mathrm{ns}}$ \\
\hline 30 & 101.9 & 151.0 & 0.7 & 1.0 & 5.9 & 5.6 & 4.1 & 3.8 & 5.4 & 4.7 & 6.9 & 7.3 \\
\hline 60 & 119.3 & 161.0 & 0.8 & 1.2 & 5.8 & 5.8 & 3.3 & 3.7 & 4.9 & 4.7 & 6.8 & 7.4 \\
\hline 90 & 114.6 & 172.3 & 0.8 & 1.4 & 6.0 & 5.7 & 3.9 & 3.4 & 5.4 & 5.1 & 7.1 & 7.1 \\
\hline 120 & 127.6 & 192.6 & 0.8 & 1.4 & 5.8 & 5.8 & 3.4 & 3.5 & 5.3 & 5.2 & 7.1 & 7.5 \\
\hline Mean & $114.1 \mathrm{~B}$ & $165.6 \mathrm{~A}$ & $0.8^{\mathrm{ns}}$ & 1.1 & $5.8^{\mathrm{ns}}$ & 5.7 & $3.7^{\mathrm{ns}}$ & 3.6 & $5.2^{\mathrm{ns}}$ & 5.1 & $6.9^{\mathrm{ns}}$ & 7.3 \\
\hline $\mathrm{CV}, \%$ & 6.8 & 18.5 & 19.5 & 14.3 & 6.5 & 5.0 & 8.3 & 11.7 & 2.8 & 5.3 & 3.8 & 6.8 \\
\hline \multicolumn{13}{|l|}{ Depth $20-40 \mathrm{~cm}$} \\
\hline 0 & $119.5^{\text {ns }}$ & $118.9^{\mathrm{ns}}$ & $1.7^{\mathrm{ns}}$ & $1.7^{\mathrm{ns}}$ & $6.1^{\text {ns }}$ & $5.5^{\mathrm{ns}}$ & $3.4^{\mathrm{ns}}$ & $3.6^{\mathrm{ns}}$ & $4.9^{\mathrm{ns}}$ & $5.1^{\text {ns }}$ & $6.6^{\mathrm{ns}}$ & $6.3^{\mathrm{ns}}$ \\
\hline 30 & 113.3 & 124.9 & 1.6 & 1.6 & 5.5 & 5.0 & 2.6 & 3.4 & 5.5 & 4.7 & 6.8 & 6.8 \\
\hline 60 & 117.2 & 125.2 & 1.6 & 1.9 & 5.5 & 5.1 & 3.1 & 3.2 & 5.1 & 4.3 & 6.7 & 6.8 \\
\hline 90 & 126.5 & 118.6 & 1.9 & 1.7 & 5.3 & 5.7 & 3.0 & 3.4 & 5.5 & 4.9 & 6.5 & 6.3 \\
\hline 120 & 117.1 & 111.0 & 1.6 & 1.5 & 4.9 & 5.2 & 2.9 & 2.4 & 5.8 & 4.8 & 6.3 & 6.2 \\
\hline Mean & $118.7^{\mathrm{ns}}$ & 119.7 & $1.7^{\mathrm{ns}}$ & 1.7 & $5.549^{\mathrm{ns}}$ & 5.3 & $3.0^{\mathrm{ns}}$ & 3.2 & $5.3^{\mathrm{ns}}$ & 4.7 & $6.6^{\mathrm{ns}}$ & 6.5 \\
\hline $\mathrm{CV}, \%$ & 16.9 & 15.4 & 12.8 & 11.9 & 8.3 & 9.1 & 27.2 & 27.9 & 3.4 & 4.7 & 13.3 & 10.2 \\
\hline \multicolumn{13}{|l|}{ Depth $40-60 \mathrm{~cm}$} \\
\hline 0 & $106.7^{\mathrm{ns}}$ & $97.8^{\mathrm{ns}}$ & $1.6^{\mathrm{ns}}$ & $1.5^{\mathrm{ns}}$ & $5.0^{\mathrm{ns}}$ & $5.3^{\text {ns }}$ & $3.1^{\mathrm{ns}}$ & $3.6^{\mathrm{ns}}$ & $5.2^{\mathrm{ns}}$ & $4.8^{\mathrm{ns}}$ & $6.3^{\text {ns }}$ & $6.9^{\mathrm{ns}}$ \\
\hline 30 & 96.0 & 99.6 & 1.3 & 1.5 & 5.6 & 5.5 & 3.1 & 3.9 & 5.1 & 5.2 & 7.0 & 7.8 \\
\hline 60 & 102.63 & 110.8 & 1.7 & 1.9 & 5.7 & 5.5 & 3.2 & 3.8 & 4.5 & 4.6 & 7.0 & 6.0 \\
\hline 90 & 106.7 & 94.8 & 1.5 & 1.6 & 6.0 & 5.3 & 2.9 & 3.6 & 4.7 & 4.9 & 7.1 & 7.1 \\
\hline 120 & 100.5 & 115.5 & 1.4 & 1.7 & 5.8 & 5.6 & 3.6 & 3.2 & 5.3 & 4.6 & 7.1 & 6.9 \\
\hline Mean & $102.5^{\mathrm{ns}}$ & 103.7 & $1.5^{\mathrm{ns}}$ & 1.6 & $5.6^{\mathrm{ns}}$ & 5.5 & $3.2^{\mathrm{ns}}$ & 3.6 & $5.0^{\mathrm{ns}}$ & 4.8 & $6.9^{\mathrm{ns}}$ & 6.9 \\
\hline $\mathrm{CV}, \%$ & 16.8 & 22.4 & 17.0 & 14.3 & 15.3 & 14.0 & 37.5 & 18.9 & 9.3 & 3.7 & 12.7 & 6.6 \\
\hline
\end{tabular}

Note. Means followed by the same letter, capital in line, do not differ each other by the Tukey's test at $\mathrm{p} \leq 0.05$; $\mathrm{ns}=$ not significant by the $\mathrm{F}$ test $(\mathrm{p} \leq 0.05) .{ }^{1} \mathrm{MBC}$, microbial biomass carbon; ${ }^{2} q \mathrm{Mic}=(\mathrm{MBC} / \mathrm{TOC}) \times 100$; ${ }^{3}$ nitrifying and ammonifying microorganisms. 

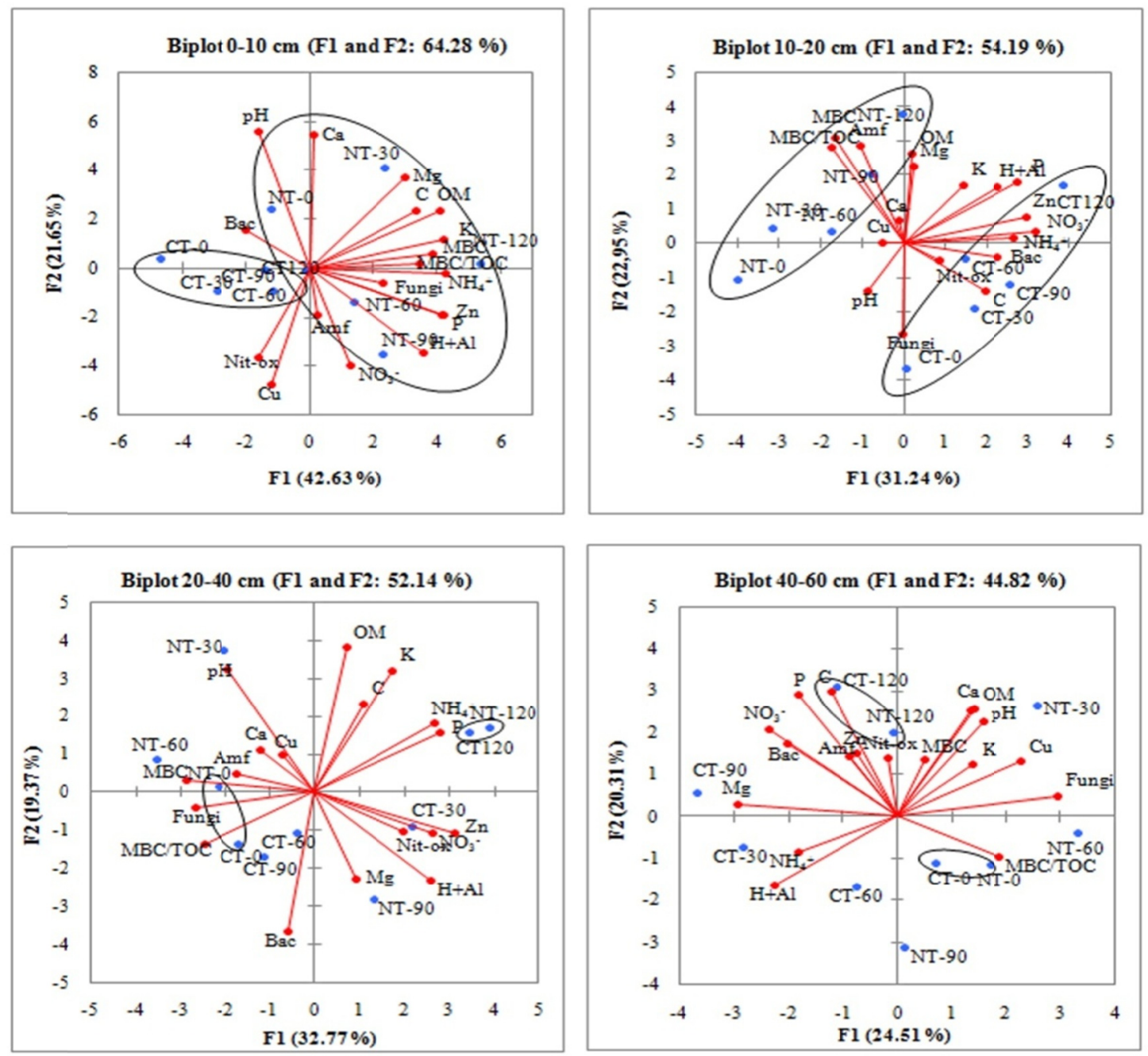

Figure 1. Principal component analysis (PCA) based on physicochemical and microbiological attributes at four depths of a clayey soil (Typic Hapludox) after applications of doses of swine wastewater (SWW) for 11 years under conventional tillage (CT) or no-tillage (NT) in southern Brazil. $\mathrm{MBC}$, microbial biomass carbon; $q \mathrm{Mic}=$ $(\mathrm{MBC} / \mathrm{TOC}) \times 100$; Bac, total bacteria; Nit-ox, nitrifying microorganisms; Amf, ammonifying microorganisms

\section{Discussion}

SWW has a high-polluting potential and must be managed properly to avoid environmental risks. Because of its high concentrations of nutrients, SWW application to the soil as source of nutrients for agricultural crops has been a rational way for its destination. However, a careful monitoring of physical, chemical and biological properties of soils receiving wastes must be done to avoid or reduce environmental risks, especially under long-term applications (Lalande et al., 2000; Scherer et al., 2010; Balota et al., 2014).

The applications of SWW for 11 years in this trial caused only minor effects on soil chemical properties, which were related to the more abundant nutrients in the residue, especially $\mathrm{N}$ and P. Effects on mineral $\mathrm{N}$ were observed only for $\mathrm{NO}_{3}{ }^{-} \mathrm{N}$ because $\mathrm{NH}_{4}{ }^{+}-\mathrm{N}$ runs fast nitrification in the soil (Aita et al., 2007). In addition to the increase of $\mathrm{NO}_{3}{ }^{-} \mathrm{N}$ with the doses of SWW at 0-10 cm and 10-20 cm, higher concentrations were found at 10-20 $\mathrm{cm}$ layer, suggesting leaching of $\mathrm{NO}_{3}^{-}-\mathrm{N}$, especially under $\mathrm{CT}$ management. Conversely, despite the increase of $\mathrm{P}$ with the doses of SWW at the two topsoil layers, higher concentrations were found at $0-10 \mathrm{~cm}$, especially under NT, because of the typical low P movement along the soil profile, especially in clayey soils. Thus, concerns on nutrient losses to water streams from soils receiving residues should consider not only leaching of $\mathrm{NO}_{3}^{-}-\mathrm{N}$ but also runoff carrying phosphates.

Despite some effect of soil management on MBC and $q$ Mic, there was no effect of the application of SWW on the microbiological attributes. This non-effect on MBC may be consequence of a rapid depletion of labile 
organic substrates provided by the SWW, which would not be enough for a long-term maintenance of the microbial development, as suggested Plaza et al. (2007). The application of 40 to $160 \mathrm{~kg} \mathrm{ha}^{-1}$ of SWW on a clayey-sandy soil (Orthic Humo-Ferric Podzol) for 5 years did not change the MBC (Carter \& Campbell, 2006), corroborating the results presented here. SWW has low $\mathrm{C} / \mathrm{N}$ ratio (Aita et al., 2007) that makes easy its mineralization in the soil. According to Guerrero et al. (2007), approximately $45 \%$ of the C added via SWW was mineralized within 16 days. In another study, with applications of 150 and $300 \mathrm{~m}^{3} \mathrm{ha}^{-1}$ year $^{-1}$ of SWW, most of the organic $\mathrm{C}$ was readily mineralized within 30 days (Plaza et al., 2007). Consequently, a long-term effect of SWW applications on $\mathrm{MBC}$ would not be expected because the $\mathrm{C}$ sources in the residue are fast mineralized and do not lead to the formation of SOM. Although MBC is a biological indicator sensitive to soil changes, its response depends on the amounts and quality of $\mathrm{C}$ compounds applied via residue and the $\mathrm{C}$ dynamics over time (Carter \& Campbell, 2006).

Effect of SWW application on the MBC would be expected closer to the application time (Guerrero et al., 2007). Increases in $\mathrm{MBC}$ were observed just after the application of SWW, reaching a maximum at 20 days, followed by a decrease up to basal levels thereafter (Guerrero et al., 2007). In another study, the addition of waste to the soil increased the MBC in the first 14-30 days after application, but the increase did not persist and gradually decreased in the following 30-120 days (Plaza et al., 2007). As the residue has low C:N ratio, it is easily degradable and has low residual effect, as can be seen by the no-effect on the TOC and SOM as well. This transient effect may explain why SWW did not affect the soil MBC in the present study, since the soil sampling was performed 44 days after the application, which is enough for mineralization of easily degradable $\mathrm{C}$ sources.

The application of SWW on a clay soil at the dose of $120 \mathrm{~m}^{3} \mathrm{ha}^{-1} \mathrm{yr}^{-1}$ for 2-3 years resulted in a microbial biomass at $0-10 \mathrm{~cm}$ of soil depth averaging 255 and $488 \mu \mathrm{g} \mathrm{g}^{-1}$ of $\mathrm{MBC}$, under conventional tillage and no-tillage system, respectively (Balota et al., 2012). In this work, which corresponds to the $11^{\text {th }}$ year on the same experimental site, there was no effect of SWW on MBC, which averaged 210 and $326 \mu \mathrm{g} \mathrm{g}^{-1}$ for the same respective soil management systems. The no-effect of SWW on MBC after 11 years of application can also be explained by the fluctuation of the soil microbial biomass due to the seasonality during the samplings. Rochette et al. (2000) reported annual fluctuation of MBC in a site receiving SWW for 19 years, being expected more discrepancies among treatments when the samples were taken during the spring-summer because of more favorable conditions of moisture and temperature for microbial growth.

The MBC decreased by $54 \%$ from $0-10 \mathrm{~cm}$ to $40-60 \mathrm{~cm}$ (Table 3), as it is associated with the levels of SOM (Ferreira et al., 2007; Balota et al., 2012). However, the conservative soil management (NT) increased the MBC at $0-10 \mathrm{~cm}$ and $10-20 \mathrm{~cm}$, in agreement with increases in the concentrations of SOC at 10-20 cm, showing that $\mathrm{MBC}$ is more responsive to soil management strategies than to applications of SWW. The PCA for 10-20 cm depth also indicates a relationship of $\mathrm{MBC}, q \mathrm{Mic}$, and ammonifying microorganisms with the soil management under NT. The maintenance of straw on the soil surface under NT increases the MBC due to increases in TOC and nutrients. Therefore, an increase of copiotrophic microorganisms rapidly mineralizes the readily available substrates, leading to an increase in the MBC that decreases thereafter with the decline of easily mineralizable organic compounds. At this stage, the microbial community is replaced by oligotrophic microorganisms, which are characterized by slow growth but are more capable of degradation of complex substrates, such as lignin, leading to the formation of OM via humification (Paul, 2007). These conditions are favored under NT mainly because of more microbial biomass and more organic substrates than in the soil under CT, in addition to more favorable conditions for microbial growth like less oscillation of temperature and moisture.

Despite the no-effect of treatments on bacterial counts, studies have shown that different soil managements change the bacterial community, like stimulation under NT system, attributed to the maintenance of soil moisture (Ferreira et al., 2017). In general, bacteria dominate the microbial community in soils under high availability of nutrients, pH close to neutral, and (often) low levels of SOM (Van Der Heijden et al., 2008), as observed in this trial, in which the bacterial density was 1.5 times higher than fungi. However, the fungal density decreased more intensively from $0-10 \mathrm{~cm}$ to $40-60 \mathrm{~cm}$ of soil depth, whereas total bacteria, nitrite oxidizers, and ammonifiers showed less intense decrease. In both cases, the decrease of SOM with depth decreases the occurrence mainly of heterotrophic microorganisms (Paul, 2007).

The $q \mathrm{Mic}$ index indicates the living part of the SOM, so that higher $q$ Mic indicates higher proportion of MBC forming the SOM, suggesting favorable conditions for microbial growth. A low $q \mathrm{Mic}$ indicates that the microbial community is under environmental stress even when the levels of SOM remain unchanged (Sparling, 1992). These results indicate that the conditions for soil microbial biomass were stressful in both SM systems, although the NT system was less stressful. Previous researches have indicated higher $q$ Mic in soil under NT $(2.2 \%)$ than in soil under CT (1.1\%) at a depth of $0-10 \mathrm{~cm}$ after 15 years of cultivation including the application of $90 \mathrm{~m}^{3} \mathrm{ha}^{-1}$ 
$\mathrm{yr}^{-1}$ of SWW (Balota et al., 2014). In another study, 14 days after the application of $300 \mathrm{~m}^{3} \mathrm{ha}^{-1}$ of SWW in a Typic Haploxeralf, the $q$ Mic reached 3.5\%, whereas in the control group it was $1.5 \%$ (Plaza et al., 2007). This is in agreement with a transient increase of MBC just after the application of SWW as discussed previously.

A clear grouping based on PCA for each SM at 0-10 cm and 10-20 cm, and an ordering of SWW doses in each $\mathrm{SM}$ at $10-20 \mathrm{~cm}$ (Figure 1) confirms that the topsoil layers are the most responsive to SM and input of residues. This is in agreement with the decrease of the $\%$ of the explained variance from $64 \%$ at $0-10 \mathrm{~cm}$ to $45 \%$ at $40-60$ $\mathrm{cm}$. However, evaluation at deeper soil layers is important for the assessment of leached ions like $\mathrm{NO}_{3}{ }^{-} \mathrm{N}$. Multivariate analysis was a useful tool to find general trends when most of the analysis of individual variables did not show statistically significant responses. For example, among the 18 variables (from which 6 biological) only few were significantly affected by the treatments. However, the global analyses by PCA allowed drawing a general trend based on the pool of variables, especially at the two topsoil layers, emphasizing the differences caused by SM and inputs of SWW, and associate them with the most affected variables.

The soil management of a clayey Typic Hapludox under NT after 11 years increased the microbial biomass and $q \mathrm{Mic}$ in the topsoil layers, but the application of increasing doses of SWW either under NT or CT caused only minor effects on chemical properties, limited to available $\mathrm{P} \mathrm{NO}_{3}^{-}-\mathrm{N}$ and $\mathrm{Zn}^{2+}$. No further effects of SM or SWW were observed on the density of bacteria, fungi, nitrite oxidizers and ammonifiers at soil depths down to $60 \mathrm{~cm}$. SM had single effects on TOC, MBC and $q$ Mic, whereas SWW had single effect only on $\mathrm{Zn}^{2+}$. Only available $\mathrm{P}$ and $\mathrm{NO}_{3}{ }^{-} \mathrm{N}$ were affected simultaneously by $\mathrm{SM}$ and $\mathrm{SWW}$. The principal component analysis based on chemical and microbiological attributes was useful to distinguish groupings between CT and NT, and SWW rates at $0-10 \mathrm{~cm}$ and $10-20 \mathrm{~cm}$, the soil layers most influenced/responsive to anthropogenic actions.

\section{Acknowledgements}

This project was financially supported by Agronomic Institute of Paraná-IAPAR, Londrina, Paraná, Brazil. We also thank the technicians Valdir L. Guerini and Jorge Felix for their assistances in the experiment in the field. M.A. Nogueira is CNPq scholar.

\section{References}

Addinsoft. (2009). XLSTAT-Base. Statistical analysis software. France: Addinsoft.

Aita, C., Giacomini, S. J., \& Hubner, A. P. (2007). Nitrificação do nitrogênio amoniacal de dejetos líquidos de suínos em solo sob sistema de plantio direto. Pesquisa Agropecuária Brasileira, 42(1), 94-102. https://doi.org/10.1590/S0100-204X2007000100013

Andrade, D. S., Miyazawa, M., \& Hamakawa, P. J. (1994). Microrganismos amonificadores e nitrificadores. In M. Hungria, \& R. S. Araujo (Eds.), Manual de Métodos Empregados em Estudos de Microbiologia Agrícola (pp. 354-367). Brasília, Brazil: EMBRAPA. Retrieved from https://www.embrapa.br/busca-de-publicacoes//publicacao/199952/Manual-de-metodos-empregados-em-estudos-de-microbiologia-agricola.

Balota, E. L., Machineski, O., \& Matos, M. A. (2012). Soil microbial biomass under different tillage and levels of applied pig slurry. Revista Brasileira de Engenharia Agrícola e Ambiental, 16(5), 487-495. https://doi.org/10.1590/S1415-43662012000500004

Balota, E. L., Machineski, O., \& Truber, P. V. (2010). Soil carbon and nitrogen mineralization caused by pig slurry application under different soil tillage systems. Pesquisa Agropecuária Brasileira, 45(5), 515-521. https://doi.org/10.1590/S0100-204X2010000500011

Balota, E. L., Machineski, O., \& Truber, P. V. (2011). Soil enzyme activities under pig slurry addition and different tillage systems. Acta Scientiarum Agronomy, 33(4), 729-737. https://doi.org/10.4025/actasciagron. v33i4.9816

Balota, E. L., Machineski, O., Hamid, K. I. A., Yada, I. F. U., Barbosa, G. M. C., Nakatani, A. S., \& Coyne, M. S. (2014). Soil microbial properties after long-term swine slurry application to conventional and no-tillage systems in Brazil. Science of the Total Environment, 490(15), 397-404. https://doi.org/10.1016/ j.scitotenv.2014.05.019

Carter, M. R., \& Campbell, A. J. (2006). Influence of tillage and liquid swine manure on productivity of a soybean-barley rotation and some properties of a fine sandy loam in Prince Edward Island. Canadian Journal of Soil Science, 86(4), 741-748. https://doi.org/10.4141/S06-007

Ceretta, C. A., Basso, C. J., Pavinato, P. S., Trentin, E. E., \& Girotto, E. (2005). Produtividade de grãos de milho, produção de matéria seca e acúmulo de nitrogênio, fósforo e potássio na rotação aveia preta/milho/nabo 
forrageiro com aplicação de dejeto líquido de suínos. Ciência Rural, 35(6), 1287-1295. https://doi.org/ 10.1590/S0103-84782005000600010

Dias-Junior, H. E., Moreira, F. M. S., Siqueira, J. O., \& Silva, R. (1998). Metais pesados, densidade e atividade microbiana em solo contaminado por rejeitos de indústria de zinco. Revista Brasileira de Ciência do Solo, 22(4), 631-640. https://doi.org/10.1590/S0100-06831998000400008

Ferreira, D. F. (2011). Sisvar: A computer statistical analysis system. Ciência e Agrotecnologia, 35(6), 1039-1042. https://doi.org/10.1590/S1413-70542014000200001

Ferreira, E. A. B., Resck, D. V. S., Gomes, A. C., \& Ramos, M. L. G. (2007). Dinâmica do carbono da biomassa microbiana em cinco épocas do ano em diferentes sistemas de manejo do solo no cerrado. Revista Brasileira de Ciência do Solo, 31(6), 1625-1635. https://doi.org/10.1590/S0100-06832007000600038

Ferreira, E. P. de B., Stone, L. F., \& Martin-Didonet, C. C. G. (2017). População e atividade microbiana do solo em sistema agroecológico de produção. Revista Ciência Agronômica, 48(1), 22-31. https://doi.org/10.5935/ 1806-6690.20170003

Goldin, A. (1987). Reassessing the use of loss-on-ignition for estimating organic matter content in noncalcareous soils. Communications in Soil Science and Plant Analysis, 18(10), 1111-1116. https://doi.org/10.1080/ 00103628709367886

Guerrero, C., Moral, R., Gómez, I., Zornora, R., \& Arcenegui, V. (2007). Microbial biomass and activity of an agricultural soil amended with the solid phase of pig slurries. Bioresource Technology, 98(17), 3259-3264. https://doi.org/10.1016/j.biortech.2006.07.015

Jahnel, M. C., Cardoso, E. J. B. N., \& Dias, C. T. S. (1999). Determinação do número mais provável de microrganismos do solo pelo método de plaqueamento por gotas. Revista Brasileira de Ciência do Solo, 23(3), 553-559. https://doi.org/10.1590/S0100-06831999000300008

Kempers, A. J., \& Zweers, A. (1986). Ammonium determination in soil extracts by the salicylate method. Communications in Soil Science and Plant Analysis, 17(7), 715-723. https://doi.org/10.1080/00103628 609367745

Lalande, R., Gagnon, B., Simard, R. R., \& Côté, D. (2000). Soil microbial biomass and enzyme activity following liquid hog manure application in a long-term field trial. Canadian Journal of Soil Science, 80(2), 263-269. https://doi.org/10.4141/S99-064

Lourenzi, C. R., Ceretta, C. A., Silva, L. S., Trentin, G., Girotto, E., Lorensi, F., \& Brunetto, G. (2011). Soil chemical properties related to acidity under successive pig slurry application. Revista Brasileira de Ciência do Solo, 35(5), 1827-1836. https://doi.org/10.1590/S0100-06832011000500037

Mercante, F. M., Francelino, C. S. F., Cavalheiro, J. C. T., \& Otsubo, A. A. (2008). Biomassa microbiana, em um Argissolo Vermelho, em diferentes coberturas vegetais, em área cultivada com mandioca. Acta Scientiarum Agronomy, 30(4), 479-485. https://doi.org/10.4025/actasciagron.v30i4.5301

Miyazawa, M., Pavan, M. A., \& Block, M. F. M. (1985). Determinação espectrofotométrica de nitrato em extratos de solo sem redução química. Pesquisa Agropecuária Brasileira, 20(1), 129-133. Retrieved from https://seer.sct.embrapa.br/index.php/pab/article/viewFile/14998/8715

Moreira, F. S., \& Siqueira, J. O. (2006). Microbiologia e Bioquímica do Solo (2nd ed., p. 729). Lavras, MG: UFLA.

Paul, E. A., \& Clark, F. E. (1996). Soil microbiology and biochemistry (2nd ed., p. 340). San Diego, Calif., USA: Academic Press.

Pavan, M. A., Bloch, M. F., Zempulski, H. C., Miyazawa, M., \& Zocoler, D. C. (1992). Manual de análise química de solo e controle de qualidade (IAPAR Circular, 76, p. 40). Londrina, Brazil: IAPAR.

Plaza, C., Garcia-Gil, J. C., \& Polo, A. (2007). Microbial activity in pig slurry-amended soils under aerobic incubation. Biodegradation, 18(2), 159-165. https://doi.org/10.1007/s10532-006-9051-0

Plaza, C., Hernández, D., Garcia-Gil, J. C., \& Polo, A. (2004). Microbial activity in pig slurry-amended soils under semiarid conditions. Soil Biology and Biochemistry, 36(10), 1577-1585. https://doi.org/10.1016/ j.soilbio.2004.07.017 
Renella, G., Chaudri, A. M., Falloon, C. M., Landi, L., Nannipieri, P., \& Brookes, P. C. (2007). Effects of Cd, Zn, or both on soil microbial biomass and activity in a clay loam soil. Biology and Fertility of Soils, 43(6), 751-758. https://doi.org/10.1007/s00374-006-0159-5

Rochette, P., Angers, D. A., \& Côté, D. (2000). Soil Carbon and Nitrogen Dynamics Following Application of Pig Slurry for the 19th Consecutive Year I. Carbon Dioxide Fluxes and Microbial Biomass Carbon. Soil Science Society of America Journal, 64(4), 1389-1395. https://doi.org/10.2136/sssaj2000.6441389x

Santos, H. G., Jacomine, P. K. T., Anjos, L. H. C., Oliveira, V. A. V., Lumbreras, J. F., Coelho, M. R., ... Oliveira, J. B. (2013). Sistema Brasileiro de Classificação de Solos (3rd ed., pp. 353). Rio de Janeiro-Brazil: EMBRAPA-Centro Nacional de Pesquisa de Solos.

Scherer, E. E., Nesi, C. N., \& Massotti, Z. (2010). Long-term swine manure fertilization and its effects on soil chemical properties in Santa Catarina, southern Brazil. Revista Brasileira de Ciência do Solo, 34(4), 1375-1383. https://doi.org/10.1590/S0100-06832010000400034

Silva, F. C. da. (2009). Manual de análises químicas de solos, plantas e fertilizantes (2nd ed., p. 627). Brasília, DF-Brazil: Embrapa Informação Tecnológica.

Soil Survey Staff. (2015). Illustrated guide to soil taxonomy (Version 2). U.S. Department of Agriculture, Natural Resources Conservation Service, National Soil Survey Center, Lincoln, Nebraska. Retrieved from https:/www.nrcs.usda.gov/wps/portal/nrcs/detail/soils/survey/class/taxonomy/?cid=nrcs142p2_053580

Sparling, G. P. (1992). Ratio of microbial biomass carbon to soil organic carbon as a sensitive indicator of changes in soil organic matter. Australian Journal of Soil Research, 30(5), 195-207. https://doi.org/ 10.1071/SR9920195

Sparling, G. P., \& West, A.W. (1998). A direct extraction method to estimate soil microbial C: Calibration in situ using microbial respiration and ${ }^{14} \mathrm{C}$ labeled sells. Soil Biology and Biochemistry, 20(3), $337-343$. https://doi.org/10.1016/0038-0717(88)90014-4

Suresh, A., Choi, H. L., \& Zhukun. (2009). Kinetics of chemical properties and microbial quantity in soil amended with raw and processed pig slurry. Asian-Australasian Journal of Animal Sciences, 22(5), $732-739$. https://doi.org/10.5713/ajas.2009.80417

Van Der Heijden, M. G. A, Bardgett, R. D., \& Straalen, N. M. V. (2008). The unseen majority: Soil microbes as drivers of plant diversity and productivity in terrestrial ecosystems. Ecology Letters, 11(3), 296-310. https://doi.org/10.1111/j.1461-0248.2007.01139.x

Vance, E. D., Brookes, P. C., \& Jenkinson, D. S. (1987). An extraction method for measuring soil microbial biomass C. Soil Biology and Biochemistry, 19(6), 703-707. https://doi.org/10.1016/0038-0717(87)90052-6

\section{Copyrights}

Copyright for this article is retained by the author (s), with first publication rights granted to the journal.

This is an open-access article distributed under the terms and conditions of the Creative Commons Attribution license (http://creativecommons.org/licenses/by/4.0/). 